\title{
Philosophiques
}

\section{À quoi peut bien servir la notion d'égalité si elle n'est ni vraiment heuristique ni simplement éristique?}

\section{Jocelyn R. Beausoleil}

Volume 11, numéro 1, avril 1984

URI : https://id.erudit.org/iderudit/203246ar

DOI : https://doi.org/10.7202/203246ar

Aller au sommaire du numéro

\section{Éditeur(s)}

Société de philosophie du Québec

\section{ISSN}

0316-2923 (imprimé)

1492-1391 (numérique)

Découvrir la revue

\section{Citer cet article}

Beausoleil, J. R. (1984). À quoi peut bien servir la notion d'égalité si elle n'est ni vraiment heuristique ni simplement éristique? Philosophiques, 11(1), 137-155. https://doi.org/10.7202/203246ar
Résumé de l'article

Je resitue tout d'abord la notion d'égalité dans le contexte historique de la société industrielle moderne, afin d'en retracer la genèse. Cette approche vise à introduire le lecteur au problème de la saisie conceptuelle de la notion d'égalité et de la détermination de son contenu. C'est l'occasion de discuter si cette notion peut répondre à une fonction heuristique ou bien si elle n'a qu'un rôle éristique. L'hypothèse développée est qu'une telle notion constitue plutôt une représentation schématique de celle de justice. La vacuité principielle du schème d'égalité $a$, par ailleurs, toujours pour effet de nous reconduire en définitive à l'examen de sa fonctionnalité opérationnelle. 


\title{
À QUOI PEUT BIEN SERVIR LA NOTION D'ÉGALITÉ SI ELLE N'EST NI VRAIMENT HEURISTIQUE NI SIMPLEMENT ÉRISTIQUE ?*
}

\author{
par Jocelyn R. Beausoleil
}

\begin{abstract}
RÉSUMÉ. Je resitue tout d'abord la notion d'égalité dans le contexte historique de la société industrielle moderne, afin d'en retracer la genèse. Cette approche vise à introduire le lecteur au problème de la saisie conceptuelle de la notion d'égalité et de la détermination de son contenu. C'est l'occasion de discuter si cette notion peut répondre à une fonction heuristique ou bien si elle n'a qu'un rôle éristique. L'hypothèse développée est qu'une telle notion constitue plutôt une représentation schématique de celle de justice. La vacuité principielle du schème d'égalité $a$, par ailleurs, toujours pour effet de nous reconduire en définitive à l'examen de sa fonctionnalité opérationnelle.
\end{abstract}

ABSTRACT. I set up at first the notion of equality within the historical context of modern industrial society, so that its genesis is recalled to mind. This way of approach aims at placing the reader in front of the problem which consists in apprehending conceptually the notion of equality and in determining its content. That gives the opportunity to inquire thereinto, if the considered notion may have an heuristic value or else only an eristic character. The unfolded hypothesis is that such a notion provides rather a schematic depiction for the one of justice. The vacuity in itself of the schema of equality compels moreover to ponder after all its operational functionality everywhere it is applied.

J'aimerais rappeler ici quelques observations faites par Raymond Aron sur le paradoxe, ou mieux encore sur la contradiction, que recèle la société industrielle moderne.

* Cette conférence fut prononcée le 25 janvier 1982 au Département de Philosophie de l'Université de Montréal, d'abord devant une équipe de recherche sur « Les théories de l'égalité et les problèmes philosophiques de la condition féminine ", et ensuite de nouveau le même jour dans le cadre d'un "Colloque sur des thèmes actuels en éthique ". Le texte de cette conférence, préalablement intitulée Le mot "égalité " peut-il vraiment correspondre à un concept beuristique ou bien n'est-il qu'un terme éristique ?, a ici été revu et augmenté pour la publication. 
Raymond Aron remarque en effet, et c'est même la thèse centrale de son ouvrage intitulé Les désillusions du progrès - Essai sur la dialectique de la modernité ${ }^{1}$, que la société industrielle moderne est à la fois égalitaire dans son aspiration et hiérarchique dans son organisation. De plus, il souligne que l'idéal égalitaire, tel que formulé au XVIII ième siècle, apparaît équivoque. Car, si l'organisation rationnelle du travail permet effectivement d'augmenter la richesse disponible pour une éventuelle réduction des inégalités, l'efficacité requise, elle, par contre, ne peut pas aller sans une certaine hiérarchie d'autorité. C'est pourquoi, conclut-il, la caractéristique fondamentale de la société industrielle moderne réside dans l'instabilité constante d'un processus. Ainsi, cette société se révèle-t-elle en elle-même essentiellement différenciée et stratifiée, en raison de la multiplicité des fonctions qu'elle comporte nécessairement. Enfin, selon Raymond Aron, l'universalisation de la société industrielle moderne engendre l'inégalité de la croissance et du progrès entre les grandes entités économico-politiques internationales, empêchant par-là même jusqu'à maintenant l'unification planétaire de l'humanité.

De ces quelques observations, je commencerais par retenir ceci : l'égalité, à laquelle se réfere le discours juridico-politique de la société industrielle moderne, renvoie à un idéal formulé au XVIII ième siècle. Il n'est nullement signifié par-là, cependant, que le mot "égalité " ne prendrait sens qu'avec la philosophie politiqùe du XVIII ième siècle. Ainsi que l'on sait, déjà dans l'Antiquité le Stoïcisme affirmait l'égalité de tous les hommes fondée sur leur commune possession de la raison. Et l'on peut dire que le Christianisme répandait lui aussi, à sa façon, l'idée d'égalité, puisqu'il professait que tous les hommes étaient égaux sous le regard de Dieu. Ce qui est plutôt signifié ici, c'est que le mot «égalité ", véhiculé dans la théorie de la société industrielle moderne, tient son sens, d'une façon à la fois plus immédiate et plus prégnante, des représentations issues d'une réflexion critique sur l'ordre social prévalant dans l'Europe aristocratique du XVIII ième siècle. C'est de cette façon que l'égalité, pour laquelle il est pris parti ou contre laquelle il est vitupéré,

1. Voir Raymond Aron, Les désillusions du progrès - Essai sur la dialectique de la modernité, Paris, "Liberté de l'Esprit " (Éditions Calmann-Lévy), 1969, xxiii/377 p. 
se comprend désormais principalement - sans que ce soit en toute exclusivité - par référence au mouvement de pensée qui se dessine de plus en plus nettement au Siècle des Lumières.

Or, ce mouvement de pensée qui donne naissance à ce sens spécifiquement moderne de l'égalité n'est pas sans équivoque. Il y a une sorte d'ambiguïté congénitale, pourrait-on dire, enveloppant dès ce moment-là le mot " égalité ». Cette ambiguïté, Raymond Aron la voit dans le fait que ce qui permettrait la mise en œuvre effective du projet d'égalité est en même temps ce qui tout aussitôt en menace la possibilité, et peut-être même la détruit. Ce à quoi Raymond Aron songe ici, c'est à l'impératif de l'organisation rationnelle de la société. En effet, l'élimination des disparités sociales suppose nécessairement une certaine efficacité qui, à son tour, requiert immanquablement une différenciation fonctionnelle. Et c'est cette même différenciation fonctionnelle qui, une fois portée à une dimension planétaire, divise l'humanité en de grands ensembles laissant voir de fortes inégalités de développement. Ainsi, le problème d'une saisie adéquate de toutes les implications théoriques de l'égalité dans la société humaine conduirait ultimement à rejoindre le problème de l'instauration d'un nouvel ordre mondial.

Je ne désirerais toutefois pas, afin de rester dans les limites de la présente communication, aller davantage de ce côté, et je préférerais plutôt m'en tenir à la question centrale de la structure de la société en tant que telle.

Il faudrait sans doute remarquer que le diagnostic posé par Raymond Aron - reconnaissant comme inéluctable une certaine hiérarchie dans la structure de l'organisation sociale - ne semble pas devoir être compris à partir de l'opposition stricte de l'homogène et de l'hétérogène. Autrement dit, si l'on observe un phénomène de hiérarchisation dans la société, cela ne provient aucunement de ce que les individus faisant partie de cette société soient absolument hétérogènes les uns aux autres. Au contraire, pour autant que la hiérarchie caractérisant la structure sociale résulte d'une différenciation fonctionnelle, cette sorte de hiérarchie n'est possible qu'à l'intérieur d'une sphère d'homogénéité englobante, dans laquelle la relation hiérarchique peut précisément se constituer et s'articuler. Je ne pense pas que, pour 
comprendre la relation hiérarchique en question, il faille faire référence à ce qui serait absolument hétérogène, à ce qui serait d'un genre tout autre, à ce qui serait l'antihomogène pur. L'on pourrait envisager théoriquement, par ailleurs, que l'opposition de l'homogène et de l'hétérogène donne lieu à une autre sorte de relation hiérarchique, mais ce ne serait pas alors une hiérarchie découlant strictement d'une différenciation fonctionnelle. Ici, suivant l'analyse que Raymond Aron propose de la société industrielle moderne, c'est bien plutôt la différenciation fonctionnelle qui, commandant la hiérarchie, produit ultimement l'inégalité dans la structure sociale. C'est donc, au fond, parce qu'il y a nécessairement une irruption de la différence dans le fonctionnement de l'organisation sociale que surgit le problème de l'inégalité.

À ce propos, l'on pourrait peut-être se demander avec Ralf Dahrendorf si l'inégalité parmi les hommes n'est pas un élément nécessaire à la structure de toute société humaine ${ }^{2}$. Cet auteur tient même le problème de l'inégalité pour la clé de l'histoire de la sociologie, en tant que cette dernière se veut justement la théorie de la société civile. C'est ainsi qu'il explique la continuité historique de cette thématique de l'égalité, qui court depuis le XVIII ième siècle jusqu'à nos jours. Selon lui, ce que le XVIIIième siècle recherchait comme l'origine de l'inégalité et ce que le $\mathrm{XIX}^{\text {ième }}$ siècle étudiait comme la formation des classes sociales, ce serait maintenant dans le cadre d'une théorie de la stratification sociale qu'il en serait traité.

Il conviendrait, cependant, de nuancer quelque peu cette thèse de Ralf Dahrendorf, car il n'apparaît pas entièrement juste de dire que les préoccupations théoriques de chacun de ces siècles aient été aussi exclusivement et aussi étroitement circonscrites les unes par rapport aux autres. Il s'agirait plutôt d'une simple question d'accentuation ou d'inflexion dans la manière de conduire l'analyse de la structure sociale. Il ne faudrait pas non plus conclure de ce survol synoptique esquissé par Ralf Dahrendorf que toute la problématique du XX $\mathrm{X}^{\mathrm{ième}}$ siècle se résume au fonc-

2. Voir Ralf Dahrendorf, "On the Origin of Inequality among Men", dans Lee Rainwater, édit., Social Problems and Public Policy - Inequality and Justice, Chicago, Aldine Publishing Company, 1974, p. 41-51. 
tionnalisme, bien qu'il y ait incontestablement une certaine " attitude fonctionnelle ", aujourd'hui assez répandue, dans la façon d'aborder les problèmes sociaux. Néanmoins, la thèse de Ralf Dahrendorf conserve un mérite indéniable, c'est celui d'attirer l'attention sur le fait que l'ambiguïté enveloppant le mot « égalité » n'est pas sans rejaillir quelque peu sur toute approche théorique de la "stratification sociale " en tant que telle. Ceci veut dire qu'une approche théorique se proposant uniquement comme «fonctionnelle " ne peut pas sans plus contourner les difficultés que suscitent la compréhension et la définition de l'égalité, et en faire ainsi l'économie la plus complète dans le cours de son analyse de la structure sociale. Ces difficultés et il nous faudra encore y revenir plus loin - tiennent à ce que la notion d'égalité se présente dans un rapport quasi synonymique à la notion d'identité.

Ainsi que tout ce qui a été précédemment exposé le laisse clairement entrevoir, la notion d'égalité - quels que soient d'ailleurs les termes variés dans lesquels elle s'énonce - comporte une genèse. Et la genèse de la notion d'égalité montre que cette notion résulte des formes de contestation qu'ont connu les structures sociales de la civilisation occidentale. Ceci est déjà la conclusion à laquelle en arrive Célestin Bouglé, dans une étude sociologique qu'il consacre aux idées égalitaires, à la toute fin du XIX ${ }^{\text {ième }}$ siècle ${ }^{3}$. Dans cet ouvrage, il insiste sur le fait que les idées égalitaires se rattachent à des jugements de valeur. Elles sont des idées morales consistant en des jugements de droit et non pas de fait. Il rappelle aussi que, pour qu'une idée s'impose à une société, il faut au moins qu'il y ait auparavant une sorte d'harmonie ou de compatibilité entre les deux. C'est pourquoi l'explication qu'il propose de la genèse de la notion d'égalité $s$ 'attache-t-elle à relever les influences et les tendances qui s'exercent dans la société occidentale, et qui conduisent peu à peu à la formation d'une telle notion. Il s'agirait donc avec l'apparition de la notion d'égalité d'un phénomène éminemment culturel, c'est-à-dire d'un phénomène lié à une certaine aire culturelle, dans la mesure où une civilisation donnée se pense ou se comprend, se définit ou se projette, à travers la notion d'égalité.

3. Voir Célestin Bouglé, Les idées égalitaires - Étude sociologique, Paris, Éditeur Félix Alcan, 1899,249 p. 
C'est encore dans cette même veine que se situe l'approche explicative développée par John W. Gardner ${ }^{4}$. Ce dernier part du fait que la plupart - pour ne pas dire l'immense majorité - des sociétés humaines à travers tous les temps ont précisément pour caractéristique fondamentale d'être des sociétés où les privilèges sont héréditaires et où la pleine réalisation des chances de chaque individu n'est pas possible. Or, l'avènement de la révolution industrielle au XVIII ${ }^{i e ̀ m e}$ siècle, précisément parce qu'il faut alors assurer la libre circulation économique, contribue à procurer une plus large autonomie à l'individu et permet le développement des idées démocratiques. Pour John W. Gardner, après l'abandon de la classification héréditaire, deux voies de développement s'offrent à la société : l'individualisme et l'égalitarisme. Ce qu'il entend ici par individualisme, c'est la compétition individuelle, c'est-à-dire la libre concurrence entre les individus. Par contre, l'égalitarisme consiste à atténuer quelque peu les différences de capacité entre les individus, de manière à ramener les chances de chacun autour d'une certaine moyenne. Il souligne, par ailleurs, que l'exaltation de la compétition peut transformer la société en une jungle d'intérêts particuliers et, parce qu'elle engendre des ambitions démesurées, provoquer du même coup d'amères désillusions. D'autre part, l'égalitarisme, s'il devient absolu, peut générer la médiocrité voire la vulgarité — du comportement, dans la mesure où il peut étouffer la lutte pour l'excellence.

John W. Gardner estime néanmoins qu'une société saine en est une où se combinent ces deux attitudes, que sont l'individualisme et l'égalitarisme. Et, dans la même foulée, il affirme en outre qu'elles correspondent à deux aspects de l'idéal américain. Cependant, il faudrait sans doute ici faire remarquer à l'encontre de cet auteur que, historiquement, la société américaine a toujours préféré la liberté à l'égalité, en ce sens que la première a toujours primé relativement à la seconde. À ce sujet, tout le problème vient selon lui de ce que, dans leurs formes extrêmes, l'individualisme et l'égalitarisme entrent en conflit et s'excluent mutuellement. C'est pourquoi, conclut-il, la tension

4. Voir John W. Gardner, Excellence - Can We be Equal and Excellent too?, New York, Harper \& Brothers Publishers, 1961, xiv/171 p. 
entre l'accentuation et la limitation de l'action individuelle est inhérente au fonctionnement le plus intime de la démocratie industrielle, et il ajoute encore qu'elle n'est même jamais parfaitement surmontable. En cela, il rejoint la thèse avancée par Raymond Aron - thèse suivant laquelle la société industrielle moderne se déploie en un processus marqué d'une constante instabilité.

Mais comment expliquer que la notion d'égalité intervienne nécessairement dans un contexte social d'instabilité et qui plus est d'instabilité constante? D'après Carl Joachim Friedrich, la notion d'égalité connaît une grande ferveur dans les situations où apparaît l'incroyance relativement aux qualités justifiant l'inégalité politique ${ }^{5}$. Or, c'est précisément là le type de situation qu'offre le XVIII ième siècle européen.

Pourtant, la notion d'égalité revêt encore un autre aspect, par lequel elle n'apparaît pas tant comme la désignation d'un but absolu en soi que comme un instrument de lutte contre les abus de la loi positive. Cette thèse intéressante est apportée par Perry Miller en rapport à la Révolution américaine de $1776^{6}$. Selon lui, dans la situation révolutionnaire en question, la notion d'égalité est intégrée à une compréhension de la loi naturelle où celle-ci est elle-même opposée à la loi effectivement donnée dans le contexte historique. L'évocation de la loi naturelle est utilisée en vue de dénoncer les abus tangibles découlant de l'ordre politique en place. Mais, comme tous les abus dénoncés ne touchent pas toujours strictement l'égalité, Perry Miller est amené à souligner l'ambiguïté que comporte - obligatoirement - la notion d'égalité dans un pareil contexte. Et il signale, de plus, les problèmes que ne manque pas dès lors de susciter la définition de l'égalité en tant qu'idéal positif, c'est-à-dire en tant qu'un idéal ayant un contenu substantiel, susceptible d'être plus ou moins cerné avec précision.

C'est pourquoi peut-on aisément rejoindre la remarque avancée par Daniel Bell, suivant laquelle la notion d'égalité n'est

5. Voir Carl Joachim Friedrich, "A Brief Discourse on the Origin of Political Equality", dans J. Roland Pennock et John W. Chapman, édit., Nomos IX : Equality, New York, Atherton Press, 1967, p. 217-227.

6. Voir Perry Miller, "Equality in the American Setting", dans Lyman Bryson et al., édit., Aspects of Human Equality, New York, Conference on Science, Philosophy and Religion in their Relation to the Democratic Way of Life Inc., 1956, p. 237-254. 
pas, dans l'Amérique du XIX ${ }^{\text {ième }}$ siècle, une notion clairement définie, et qu'on se réfere plutôt alors à l'absence de déférence sociale : "What it meant, in effect, was that no one should put on the airs of an aristocrat and lord it over other men. To this extent, it was a negative reaction to the highly mannered society of Europe, and travelers to the United States understood it in those terms." à l'absence de déférence sociale spécialement dans le sens anglosaxon de l'expression "social deference", pour en bien saisir la portée très restreinte. D'un autre côté, il est permis d'énoncer - sans grand risque de se tromper - que cette remarque formulée par Daniel Bell en se rapportant à la situation sociale du $\mathrm{XIX}^{\text {ième }}$ siècle américain peut encore caractériser la situation que nous vivons aujourd'hui. Car, s'il est une notion de l'égalité largement répandue qui soit spontanément appuyée par la grande majorité de la population, c'est bien celle de l'absence de toute déférence sociale manifeste au sein de relations protocolaires. Or, cette simple attitude dans le comportement, que devient alors l'égalité, ne peut se comprendre qu'à partir du mouvement de rejet d'une organisation sociale axée sur les privilèges du rang que confere la noblesse. C'est en ce sens que la notion d'égalité en vient à se confondre avec celle de démocratie, dans la mesure où la démocratie se veut justement la négation - ou parfois encore la destruction - de tout ordre social à caractère aristocratique, et même à la limite de tout élitisme. Toutefois, ainsi que nous l'avons déjà à maintes reprises rappelé, la question de l'égalité n'est, en réalité, jamais aussi simple. Et, de ce point de vue, la remarque de Daniel Bell garde sans doute le mérite d'inciter à penser qu'il y aurait peut-être une distinction à établir entre une forme globale d'égalité caractérisant les relations sociales et une autre forme plus spécifique d'égalité touchant les rapports économico-politiques à l'intérieur d'une société.

En fait, il faut reconnaître qu'il est extrêmement difficile de se représenter ou même d'imaginer une société reposant effec-

7. Daniel Bell, "A 'just' Equality", Dialogue (Washington, D.C.), vol. 8, 1975, no 2, p. 85 (trad. franç. : " Ce qu'elle [c'est-à-dire la notion d'égalité] signifiait, en effer, c'était que personne ne pouvait se donner les airs d'un aristocrate et en imposer aux autres hommes. Dans cette mesure, elle était une réaction négative à la société européenne aux manières prétentieuses, et des voyageurs aux États-Unis [par exemple Alexis de Tocqueville] l'ont comprise en ces termes. "). 
tivement sur les préceptes de l'égalité la plus absolue, et qui aussi soit vraiment fonctionnelle. Je renverrai ici simplement à une remarque d'André Béteille qui rappelle que toute société est inconcevable sans conventions et sans règles, parce que toute société en tant qu'ensemble d'individus en interaction requiert nécessairement un certain degré d'organisation impliquant une distribution du pouvoir ${ }^{8}$. D'aucuns pourraient objecter ici que, même si une distribution du pouvoir est inévitable à l'intérieur de l'organisation sociale, il ne s'ensuit pas nécessairement qu'une telle distribution doive être inégale. En outre, si certaines utopies ne vont pas jusqu'à envisager une distribution du pouvoir qui soit parfaitement égale entre tous les membres de la communauté, du moins prévoient-elles une rotation rituelle dans l'exercice de la charge suprême. Seulement, le fait que toutes ces utopies n'aient jamais pu fonctionner adéquatement vient pertinemment renforcer la thèse énoncée par André Béteille. Lorsque, par contre, l'on pose son regard sur la société réelle et que l'on en observe le fonctionnement concret, l'on est bien contraint d'admettre que tout exercice du pouvoir suppose, à la limite de son principe même, une différence de force. La nature de cette différence de force peut, par ailleurs, être éminemment variable, dépendamment du degré de développement culturel atteint par une société donnée.

En effet, deux forces d'égale puissance ne pouvant jamais que s'annuler, la mise en mouvement qu'est l'exercice du pouvoir requiert fondamentalement qu'il y ait quelque part un excédent différentiel. L'on voit donc par-là que c'est la société en ellemême qui, de par sa structure constitutive, génère l'inégalité — plus précisément - l'inégalité politique. André Béteille est d'ailleurs amené à dénoncer le caractère purement mythique de la "société égalitaire primitive ". Cependant, et ceci est plus intéressant pour notre propos, il est également conduit à attirer l'attention sur le fait que la société industrielle moderne, tout en atténuant certaines anciennes formes d'inégalité, en fait surgir de nouvelles. Ceci veut dire que, même si la mise en œuvre des idées démocratiques élaborées depuis le XVIII ième siècle a effec-

8. Voir André Béteille, Inequality among Men, Oxford, "Pavilion Series - Social Anthropology" (Basil Blackwell), 1977, x/178 p. 
tivement réduit les inégalités prévalant dans l'ordre social antérieur, l'on n'a pas encore atteint pour autant l'égalité, telle que l'on peut s'en faire une représentation dans la pureté de son idée.

Mais cette "égalité ", pouvons-nous vraiment savoir ce qu'elle est ou - plutôt - pouvons-nous tout au moins la pressentir dans ce qu'elle devrait être ? Il faut ici convenir que cette question touchant l'appréhension conceptuelle de l'égalité soulève un problème méthodologique d'importance capitale. Car, pour pouvoir dire d'une société en constante évolution qu'elle s'approche de l'égalité ou qu'au contraire elle s'en éloigne, il faut que nous disposions au préalable d'une notion de l'égalité qui soit à la fois parfaitement claire et pleinement satisfaisante - parfaitement claire dans la représentation que nous en avons et pleinement satisfaisante dans l'application que nous en pouvons faire. Le problème consiste dès lors à déterminer l'égalité suivant une notion qui puisse se prêter à un traitement efficace, c'est-à-dire qui puisse entrer dans une opération concluante. Et c'est en ce sens qu'on doit se demander si le mot "égalité " peut vraiment correspondre à un concept heuristique, c'est-àdire à un concept qui soit conducteur de connaissance dans le champ de la théorie de la société.

Nous devons admettre qu'une telle question n'est pas sans susciter des difficultés particulières, compte tenu de ce que la genèse de la notion d'égalité, telle que nous l'avons précédemment mise au jour, n'obéit nullement à une motivation heuristique. Cependant, nous pouvons malgré tout, pour le moment, tenter de délimiter le champ sémantique de la notion d'égalité. À ce propos, Hugo Adam Bedau remarque que l'égalité se dit en référence à des choses qui ne sont pas identiques, mais qui "sont néanmoins en quelque manière " le "même " (the same), bien qu'elles " ne soient pas exactement "le "même " ${ }^{9}$. Les formulations tortueuses, auxquelles cet auteur se voit dans l'obligation de recourir, traduisent déjà très bien, à elles seules, tout l'inconfort intellectuel attaché à une entreprise de définition de l'égalité. La conclusion qu'il en tire finalement, c'est que l'éga-

9. Voir Hugo Adam Bedau, "Egalitarianism and the Idea of Equality", dans J. Roland Pennock et John W. Chapman, édit., Nomos IX : Equality, New York, Atherton Press, 1967, p. 3-27. 
lité, loin d'impliquer l'identité, impliquerait plutôt une certaine non-identité.

C'est aussi la position qu'exprime Marc Vanquickenborne, lorsqu'il rappelle que l'égalité ne se dit jamais que sous un certain rapport, et que, partant, elle suppose une certaine non-identité, puisque là où il y a identité stricte il ne saurait être question d'égalité $^{10}$. Néanmoins, il faut noter que, au plan théorique, toute distinction de l'identité et de l'égalité pose de sérieux problèmes, tant au niveau simplement terminologique qu'au niveau strictement logique. Au niveau terminologique, d'abord, il y a incontestablement une certaine parenté lexicale entre les deux mots, qui apparaît peut-être moins clairement en français et en anglais, mais qui ressort très nettement dans d'autres langues, par exemple en allemand où " die Gleichheit "signifie à la fois l'identité (the identity) et l'égalité (the equality). Au niveau logique, ensuite, il y a assûrément une quelconque proximité conceptuelle entre les deux mots, puisque l'on est sans cesse renvoyé de l'un à l'autre dans toute tentative de les définir, comme si leurs significations respectives passaient insensiblement l'une dans l'autre. Par ailleurs, comme le dit Marc Vanquickenborne, il est bien évident que si l'identité et l'égalité signifiaient rigoureusement la même chose il n'y aurait plus aucun intérêt à parler d'égalité. L'identité absolue rendrait inutile ou — plus exactement - superflue toute référence à l'égalité, dans la mesure où l'égalité serait toujours déjà incluse dans une telle identité.

D'une part, donc, le discours de l'égalité - c'est-à-dire le discours sur et/ou pour l'égalité - suppose, pour être possible, une certaine non-identité. Mais, d'autre part, si l'égalité doit pouvoir se dire sous un certain rapport, elle nécessite - précisément sous le rapport en question - une forme d'identité au moins partielle ou relative. L'identité partielle ou relative, c'est cette forme d'identité que l'on trouve entre deux ensembles ayant au moins un élément ou un membre commun aux deux, c'est-à-dire quelque chose apparaissant identiquement chez l'un et chez l'autre, alors que tout le reste est différent. Ainsi, bien

10. Voir Marc Vanquickenborne, «La structure de la notion d'égalité en droit ", Revue internationale de philosophie, vol. 25, 1971, no 97, fasc. 3, p. 331-350. 
que l'égalité ne puisse pas être définie en tant qu'identité, il appert qu'elle ne peut pas non plus être pensée sans référence à l'identité en général. La notion d'identité se rapporterait à la notion d'égalité, un peu comme l'horizon appartient nécessairement à tout paysage. En ce sens, la dissociation de l'identité et de l'égalité garderait toujours en définitive, au plan théorique, un caractère éminemment artificiel et irrémédiablement fragile, pour autant que la notion d'égalité est constamment rappelée dans le champ d'attraction qu'exerce la notion d'identité. Et c'est probablement là ce qui incite Marc Vanquickenborne à ne voir finalement dans l'égalité qu'une notion pragmatique. Ce que cet auteur entend par une notion pragmatique, c'est une notion dont l'application requiert simplement la référence à une certaine équivalence déterminée. Ceci voudrait dire, par conséquent, qu'il est ultimement vain de chercher un fondement théorique à la notion d'égalité en tant que telle, puisque celleci trouverait toute la justification qui lui est nécessaire uniquement dans son applicabilité.

Pourtant, tout en n'étant qu'une notion pragmatique, la notion d'égalité présente des paradoxes dans son application. Et ces paradoxes ressortent avec d'autant plus de clarté, lorsque l'on tente d'introduire certaines distinctions à l'intérieur même de la notion d'égalité. Ainsi peut-on distinguer entre "l'égalité devant la loi " qui consiste dans l'égalité formelle, c'est-à-dire dans ce que l'on appelle la régularité ou la sécurité juridiques, et « l'égalité dans la loi " qui consiste dans l'égalité réelle ou matérielle, c'est-à-dire dans l'égalité en tant qu'objectif ou but à atteindre socialement. À ce propos, Léon Ingber remarque que l'application de la première conduit le plus souvent à l'aggravation des inégalités de fait, alors que l'application de la seconde, en poursuivant une plus grande égalisation des conditions de vie, recourt à des inégalités de traitement ${ }^{11}$. Il semblerait dès lors que la notion d'égalité, bien qu'elle soit une idée-force de la société industrielle moderne, ne puisse que devenir de moins en moins claire, au fur et à mesure que s'étend son champ d'application. Parvenant à cette conclusion, Léon Ingber est conduit

11. Voir Léon Ingber, "L'égalité en droit ou le droit à l'égalité ", Journal des tribunaux (Bruxelles, Éditeurs Maison Ferdinand Larcier S.A.), vol. 94, no 5082, 12 mai 1979, p. 313-320. 
à reconnaître que la notion d'égalité cesse en fait d'être liée à une conception mathématique, et qu'elle devient plutôt fonction des valeurs ayant cours dans la société.

Ceci nous amène à la question posée par Henri Buch, lorsqu'il se demande si l'égalité est une notion statique ou bien si elle n'obéit pas plutôt à une conception évolutive ${ }^{12}$. Après avoir examiné la question, il penche pour la thèse voulant que la représentation de l'égalité soit intimement liée aux représentations philosophiques fondamentales d'une société, et que, donc, elle soit elle-même engagée dans le processus plus général de l'évolution des mentalités. Cependant, j'aimerais attirer ici l'attention sur ce que pareille thèse peut comporter - tout à la fois - de pertinent et d'insolite. Ce qu'elle peut comporter de pertinent, cela nous est devenu déjà suffisamment présent à l'esprit, lorsque nous avons tenté une saisie conceptuelle rigoureuse de ce qu'est l'égalité. La notion théorique de l'égalité nous est alors apparue difficilement cernable, très inconsistante, presqu'évanescente. Par contre, ne semble-t-il pas à première vue insolite de parler, ainsi que le suggère Henri Buch, d'un " concept d'égalité » qui puisse être, non pas statique, mais bien évolutif. N'y a-t-il pas là quelque chose qui soit propre à étonner quiconque se situe dans une approche analytique de la notion d'égalité, prise en elle-même et pour elle-même ? L'égalité n'étant toujours que ce qui peut être - par excellence - égal à soimême, c'est-à-dire égal quant à sa teneur conceptuelle, il apparaîtrait, tout au moins au premier abord, que l'égalité ne puisse jamais être qu'en tant que constamment elle-même. Le fait que la notion d'égalité ne soit pas employée partout dans un même sens ou encore le fait qu'elle soit à chaque fois spécifiée dans un rapport déterminé à des objets, tout ceci ne change strictement rien à la question, puisqu'il demeure en fin de compte que l'on doit définir l'identité conceptuelle de la notion d'égalité, faisant seule que l'on sait ce qu'on dit quand on parle d'égalité.

Je désirerais, à ce moment-ci, proposer une autre perspective qui, tout en s'écartant de la thèse défendue par Henri Buch, puisse néanmoins intégrer tout ce que nous avons jusqu'à main-

12. Voir Henri Buch, "La notion d'égalité dans les principes généraux du droit ", Revue internationale de philosophie, vol. 25, 1971, no 97, fasc. 3, p. 351-380. 
tenant appris sur la notion d'égalité. Il est possible, selon cette nouvelle perspective, de concevoir l'égalité comme étant bel et bien constamment elle-même. Mais ceci n'implique aucunement, comme semble le croire Henri Buch, que l'égalité soit alors conçue suivant une notion exclusivement statique. Et il ne s'agit pas davantage, à cette occasion, de voir dans l'égalité une notion demeurant inexorablement identique à elle-même, alors qu'elle est engagée dans un devenir évolutif conçu de manière dialectico-spéculative. Pour la perspective ici proposée, l'égalité est constamment l'égalité, justement en raison de ce qu'elle est fondamentalement vide. Cependant, si nous disons de l'égalité qu'elle est vide, ce n'est pas que l'égalité ne veuille radicalement rien dire, ni non plus qu'elle ne revête absolument aucune signification. La vacuité en question est une vacuité touchant le contenu positif de la notion d'égalité. Par-là rejoint-on, finalement, la thèse que développe Gerhard Leibholz, lorsqu'il énonce que la substance matérielle du concept d'égalité n'est que de validité relative et qu'elle est changeante quant au contenu ${ }^{13}$. Ceci indique clairement comment l'égalité ne peut jamais avoir de contenu qui lui soit strictement propre, et, aussi, comment l'égalité peut indéfiniment servir de vêtement d'emprunt à des contenus toujours autres et nouveaux. Toutefois, il importe ici de bien comprendre que ce n'est pas parce que l'on constate, d'abord, que la notion d'égalité fluctue culturellement que l'on en conclut, après coup, à sa vacuité conceptuelle. C'est, au contraire, parce qu'il y a une vacuité fondamentale - principielle - de la notion d'égalité que la fluctuation culturelle est ensuite rendue possible. Et, sous ce rapport, l'on peut, me semble-t-il, différencier la thèse avancée par Henri Buch de celle esquissée par Gerhard Leibholz, dans la mesure où la première s'en tient à une constatation simplement empirique, alors que la seconde comporte ce que l'on pourrait peut-être appeler une préoccupation fondationnelle.

Reprenant à mon compte cette préoccupation fondationnelle, je serais tenté de dire que c'est justement cette vacuité principielle de la notion d'égalité qui en fait une notion pouvant

13. Voir Gerhard Leibholz, Die Gleichbeit vor dem Gesetz - Eine Studie auf rechtsvergleichender und rechtsphilosopbischer Grundlage, Berlin, Verlag von Otto Liebman, 1925, 161 p. 
éventuellement jouer le rôle d'un terme éristique, c'est-à-dire pouvant fonctionner comme un terme central dans le discours de la controverse, de la protestation, de la revendication. Il est bien sûr, par ailleurs, que la notion d'égalité ne se réduit jamais tout à fait à un terme qui soit essentiellement et exclusivement éristique. Mais, parce que le mot "égalité » ne peut pas avoir - et cela au principe même - de contenu qui soit vraiment précis, il peut lui arriver de n'avoir qu'une portée simplement polémique, plutôt que réellement théorique. Et de là viendrait aussi, suivant les observations faites par Stanley I. Benn et R.S. Peters dans The Principles of Political Thought, que les revendications pour l'égalité prennent une forme négative, en ce sens qu'elles ne sont que des dénégations de la légitimité de certaines inégalités existantes ${ }^{14}$. Il ne s'agirait donc pas d'atteindre à une « égalité » idéale, telle qu'on pourrait en donner une représentation théorique et universelle. Le mouvement de l'égalité, c'està-dire le mouvement en faveur de l'égalité et pour l'extension de son applicabilité, conduirait seulement à éliminer des distinctions maintenant jugées injustifiables, alors que de nouvelles distinctions tenues pour acceptables seraient instaurées. Voici comment Stanley I. Benn présente, succinctement, la chose : "Equality very rarely means treating everyone alike; usually it means getting rid of one system of distinctions and replacing it with another." ${ }^{15}$ L'égalité apparaîtrait dès lors comme engagée dans un processus de substitution où un système de distinctions sociales donné est susceptible d'être remplacé par un autre système de distinctions sociales - et cela indéfiniment.

Or, la notion d'égalité ne peut se prêter à un tel processus qu'à la condition de ne receler par elle-même que très peu de densité conceptuelle, et de ne consister qu'en une pure configuration schématique, dans laquelle trouve à se loger une substance conceptuelle sans cesse renouvelée par le flux de circulation des représentations. Ainsi comprise, la notion d'égalité

14. Voir Stanley I. Benn et R.S. Peters, The Principles of Political Thought, New York, First Free Press Paperback Edition, 1965, 478 p.

15. Stanley I. Benn, "Equality, Moral and Social", dans Paul Edwards, édit. en chef, The Encyclopedia of Pbilosopby (New York and London, Crowell, Collier and Macmillan Inc., 1967), vol III, p. $41 \mathrm{~b}$ (trad. franç. : " L'égalité veut très rarement dire que l'on traite tout le monde de la même manière ; habituellement, cela veut dire que l'on se débarrasse d'un système de distinctions et qu'on le remplace par un autre. "). 
s'apparenterait à quelque chose ressemblant à un schème, dans la mesure où elle fonctionnerait comme un procédé visant à procurer la représentation schématique, c'est-à-dire l'image d'une autre représentation ou d'une certaine constellation de représentations ${ }^{16}$. En ce sens, la représentation de l'égalité interviendrait dans le discours pour imager quelque chose d'autre qu'elle-même et permettre ainsi de le visualiser plus sensiblement.

Mais de quoi d'autre pourrait-il donc s'agir alors ? À cette question, une citation de Chaïm Perelman apporte un commencement de réponse : " Alors que la notion de justice semble l'une des plus controversées qui soient, puisqu'il est normal de voir chacune des deux parties, dans les conflits qui les opposent, prétendre que c'est sa cause qui seule est juste, la notion d'égalité est susceptible d'une définition formelle et incontestée. Il est tentant de mettre les deux notions en rapport, et d'éclairer l'une d'entre elles par l'autre. " ${ }^{17}$ Dans le couple de notions formé de la « justice » et de l' "égalité », la notion de justice apparaîtrait comme celle qui est à éclairer, alors que la notion d'égalité se voudrait celle qui est éclairante, ou tout au moins qui devrait l'être. Or, l'analyse de la notion d'égalité nous a menés à la constatation que, au plan théorique, cette notion ne peut livrer en fait qu'une lumière fort parcimonieuse sur la nature de la justice - tellement parcimonieuse même qu'elle risque plutôt de l'obscurcir. En effet, dans la mesure où la notion d'égalité

16. Je me réfere ici à la définition du schème que donne Immanuel Kant dans La Critique de la raison pure: "Diese Vorstellung nun von einem allgemeinen Verfahren der Einbildungskraft, einem Begriff sein Bild zu verschaffen, nenne ich das Schema zu diesem Begriffe. " (Immanuel Kant, Kritik der reinen Vernunft, dans Gesammelte Schriften, herausgegeben von der Königlich Preussischen Akademie der Wissenschaften, Berlin, Druck und Verlag von Georg Reimer, 1911 : Text der Ersten Auflage 1781, Band IV, p. 100101 ; Text der Zweiten Auflage 1787, Band III, p. 135 (trad. franç. : « Cette représentation, donc, d'un procédé général de l'imagination pour procurer à un concept son image, je l'appelle le schème de ce concept. ")). Ce que je retiens de la définition kantienne du schème, c'est cette idée d'une représentation servant d'image à une autre représentation. Bien entendu, je n'entends pas le moindrement faire de la représentation de l'égalité le produit de l'imagination transcendantale, ni non plus rapporter une telle représentation au schématisme de l'entendement. Tout ce que je me propose par cette référence à Immanuel Kant, c'est d'utiliser une définition du schème qui m'apparaît propre à illustrer notre propos.

17. Chaïm Perelman, "Égalité et justice ", dans L'Égalité - Travaux du Centre de Philosophie du Droit de l'Université Libre de Bruxelles (Bruxelles, Établissements Émile Bruylant), vol. V, 1977 , p. 324. 
demeure une notion dont la teneur conceptuelle n'est que de clarté apparente, la tentative de définir la substance de la justice par la seule notion d'égalité se révèle, en définitive, susceptible d'égarer.

C'est pourquoi, même si elle peut sembler une notion pragmatique (au sens donné à ce terme par Marc Vanquickenborne), la notion d'égalité ne peut pas raisonnablement donner lieu à une règle morale positive, c'est-à-dire à une règle qui soit explicitement formulée dans un ensemble de propositions et qui puisse toujours être conséquemment appliquée sans jamais tomber dans les paradoxes précédemment rappelés par Léon Ingber. En disant cela, je désirerais rejoindre ici la remarque très opportune de Monroe C. Beardsley, pour qui "l'injonction d'égalité " l'idée de faire de l'égalité un précepte - n'est pas en soi une règle morale positive, mais bien plutôt une règle métaéthique ${ }^{18}$. Ainsi que nous l'avons vu, la notion d'égalité n'a pas de contenu substantiel inhérent, qui soit à la fois suffisamment significatif par lui-même et stable. Et c'est d'ailleurs ce qui fait qu'elle n'opère qu'à titre de représentation schématique. Cependant, cette simple représentation schématique ne peut en aucun cas prescrire l'obligation stricte de traiter effectivement tout le monde également en toutes circonstances. Tout ce qu'elle présuppose, c'est l'exigence logique de légitimer en regard de la justice tout traitement qui s'écarterait de la représentation de l'égalité. L'idée de faire de l'égalité un précepte est donc métaéthique, en ce sens qu'elle exprime le schème de la loi morale, c'est-à-dire plus généralement la représentation schématique du principe constitutif de toute loi.

Ayant tenté de jeter quelque lumière sur les difficultés que suscite tout effort de conceptualiser l'égalité, il nous apparaît que, dans l'ordre de la théorie de la société, la notion d'égalité n'a pas de fonction vraiment heuristique, ni qu'elle ne se réduit, non plus, à n'être que simplement éristique. Par contre, pour pouvoir en approfondir véritablement le sens, nous devons envisager d'ouvrir une certaine perspective sur la théorie du droit.

18. Voir Monroe C. Beardsley, "Equality and Obedience to Law", dans Sydney Hook, édit., Law and Pbilosophy - A Symposium, New York, New York University Press, 1964, p. 3542. 
Et nous y sommes d'ailleurs conduits par suite de ce que la représentation de l'égalité appartient en tant que schème au tissu constitutif de la loi elle-même. C'est ce que nous rappelle encore Vittorio Mathieu en ces termes : « L'eguaglianza giuridica, nella sua forma essenziale, è una proprietà che la legge possiede per il fatto stesso d'essere una prescrizione generale : almeno nella sua forma, se non nella sostanza. " ${ }^{19}$ Le droit consiste en effet en un système de prescriptions générales, c'est-à-dire en un ensemble de propositions, dans lesquelles s'exprime l'obligation juridique. Or, toute proposition exprimant l'obligation juridique comporte - de manière explicite ou implicite - un opérateur que l'on peut définir comme un quantificateur universel. Ainsi, pour la théorie du droit, l'égalité juridique ne signifie rien d'autre que le principe de l'universalité. Mais ceci, encore une fois, n'est possible que parce que, en elle-même, la notion d'égalité ne renvoie jamais à rien de singulier, à rien de particulier, à rien de spécifique, et qu'elle reste toujours et nécessairement de l'ordre général du schème.

Dès lors, on ne peut se dérober à la question de savoir si la notion d'égalité constitue le schème adéquat de l'idée de justice. Selon Julien Freund, qui s'intéresse également à ce problème des relations entre la justice et l'égalité, la première ne s'épuise pas exclusivement dans la seconde ${ }^{20}$. Il rappelle à cet effet une distinction, qui lui apparaît primordiale, entre l'égalité et la justice. L'égalité vise chaque fois à établir une équivalence entre des choses sous un rapport déterminé, c'est-à-dire qu'elle a la nature d'une convention et qu'elle est d'ordre rationnel statique. Au contraire, la justice concerne fondamentalement la conduite des êtres, et elle ne se limite donc pas à définir une correspondance rigoureuse qui serait de caractère proportionnel. Or, c'est précisément parce qu'il y a une impossibilité de tout régler par l'égalité que la justice demeure indispensable - et ce par-delà même l'égalité. Ceci veut dire que, là où l'égalité ne peut rigoureusement plus intervenir, il appartient à la justice

19. Vittorio Mathieu, "L'eguaglianza giuridica ", Rivista internazionale di filosofia del diritto, vol. 54, 1977, p. 19 (trad. franç. : " L'égalité juridique, dans sa forme essentielle, est une propriété que la loi possède de par le fait même d'être une prescription générale : au moins dans sa forme, si ce n'est dans la substance. ».)

20. Voir Julien Freund, "Justice et égalité », Les études philosophiques, vol. 28, 1973, p. 165177. 
de trouver une forme d'équilibre convenable. Autrement dit, pour reprendre la conclusion à laquelle en arrive Julien Freund, la justice n'est pas qu'égalitaire, alors que, par ailleurs, il se peut que l'égalité comporte des injustices. C'est pourquoi, enfin, est-il conduit à dénoncer l'égalitarisme - que l'on pourrait qualifier de doctrinaire et d'inconditionnel - qui croit pouvoir subordonner tout rapport à un rapport d'égalité unique et universel. Afin de contrer ce qu'il estime être une dégradation et une falsification de la justice, Julien Freund maintient pour l'idée de justice un fondement éthique irréductible à la représentation que peut en donner la notion d'égalité.

En ce sens, le recours à la notion d'égalité - l'utilisation de cette sorte de schème technique d'intervention juridico-sociale - doit rester toujours ordonné à une vision de la justice et être constamment guidé par elle. Il m'apparaît aussi, à la suite de Julien Freund, que la justice est structurellement nécessaire à la cohésion de la société humaine. En effet, une certaine fonctionnalité minimale de cette société est dangereusement menacée, si ne sont pas prises en compte les exigences de justice les plus fondamentales, faisant seules que les individus soient réellement intéressés à participer au maintien d'une telle société.

Département de philosophie

Université de Montréal 